\title{
Stress analysis of different configurations of 3 implants to support a fixed prosthesis in an edentulous jaw
}

João Paulo da Silva-Neto(a) Marcele Jardim Pimentel(a) Flávio Domingues das Neves ${ }^{(b)}$ Rafael Leonardo Xediek Consani(a) Mateus Bertolini Fernandes dos Santos ${ }^{(a)}$

(a) Department of Prosthodontics and Periodontology, Piracicaba Dental School, Universidade Estadual de Campinas Unicamp, Piracicaba, SP, Brazil.

(b) Department of Occlusion, Fixed Prostheses, and Dental Materials, Uberlândia School of Dentistry, Universidade Federal de Uberlândia - UFU, Uberlândia, MG, Brazil.
Abstract: This study's aims was to evaluate the stress distribution in a mandibular implant-supported prosthesis and peri-implant bone considering implant quantity, diameter and position using linear 3-D finite element analysis. Models of an anterior jaw comprised 4 groups according to implant quantity, diameter, and position: control group C, 5 regular implants; R, 3 regular implants; W, 3 wide implants; and DTR, 3 regular implants with the distal ones tilted $30^{\circ}$ distally. The cantilever was loaded with an axial load of $50 \mathrm{~N}$. Data was evaluated using von Mises stress on implants and maximum principal stress and microstrain on the bone. The W group showed the lowest value of maximum principal stress in peri-implant bone of the loaded side $(4.64 \mathrm{MPa})$ when compared to C (5.27 MPa), DTR (5.94 MPa), and R (11.12 MPa). Lower stress values in the loaded implants were observed in the experimental groups when compared to the $\mathrm{C}$ group. However, the unloaded implants presented opposite results. All the screws of the $\mathrm{W}$ group presented lower stress values when compared to the $\mathrm{C}$ group. However, the R and DTR groups presented an increase in stress values with the exception of the loaded screw. A reduction in the number of implants associated with wider implants reduced the stress in the bone and prosthetic components.

Descriptors: Dental Implants; Jaw, Edentulous; Finite Element Analysis.

\section{Introduction}

Fixed implant-supported prostheses have shown clinical success and proven functional benefits for use in the edentulous mandible. ${ }^{1,2}$ Changes in the original protocol have been proposed to simplify and optimize the rehabilitation of edentulous patients, increasing access to treatment. ${ }^{3-9}$ Branemark et al. ${ }^{3}$ initially proposed the use of 3 wide implants to support a fixed implant-supported prosthesis in the edentulous mandible, obtaining a success rate of $98 \%$ over 3 years of follow-up. However, this rehabilitation did not achieve similar results compared to conventional implant-support rehabilitations with immediate loading. ${ }^{3,8,10}$ Consequently, this therapy is not often prescribed. The unfavorable results were attributed to the angulation of the implants during the surgical procedure and the difficulty in obtaining a passive fit with the prefabricated metallic frameworks. ${ }^{8,9}$

Based on the Novum protocol, Branemark ${ }^{3}$ developed a new proposal
Submitted: May 07, 2013

Accepted for publication: Aug 12, 2013

Last revision: Sep 03, 2013

Braz Oral Res., (São Paulo) 2014;28(1):1-7 
for a complete fixed mandibular implant-supported prosthesis. This option aimed to provide a less expensive fixed implant-supported prosthesis and a simplified surgical procedure, which could be applied to a broader range of edentulous patients. This new concept of prosthetic rehabilitation consists of a complete fixed mandibular prosthesis supported by 3 implants splinted with a manufactured metal framework, subjected to immediate loading. Implants are placed between the mental foramina. Two distal implants are placed next to the foramina, and a single central implant is placed at the midline. ${ }^{9}$ The use of 3 regular implants in this configuration has shown promising clinical results, with success rates above $96 \%{ }^{8,9}$ However, data on the limitations and long-term complications of these rehabilitations are still insufficient. ${ }^{8,9}$

Increasing the implant diameter and tilting the distal implants have been proposed to improve the biomechanical behavior by increasing the contact surface area and reducing the average cantilever length of the prosthesis. ${ }^{5-7,11,12}$ However, it is unclear how the number, diameter, and arrangement of the implants impact the biomechanical behavior of fixed implant-supported complete dentures. Given the absence of experimental studies that support the clinical use of this configuration, the aim of this study was to use linear three-dimensional (3D) finite element (FE) analysis to evaluate the stress distribution in the peri-implant bone and prosthetic components with different implant configurations. The hypothesis was that the stress distribution in a fixed implant-supported prosthesis is influenced by the number, diameter, and arrangement of implants.

\section{Methodology}

A 3D modeling software (SolidWorks 2010, SolidWorks Corp., Concord, USA) was used to build a model of a severely resorbed jaw, with external hex titanium implants (10.0 mm length) between the mental foramens and a fixed implant-supported prosthesis seated on the implants by UCLA abutments and a titanium metal bar (5.0 $\mathrm{mm}$ diameter). Models were divided into 4 groups according to the number, diameter, and arrangement of the implants.
Table 1 - Material properties adopted in this study.

\begin{tabular}{c|c|c}
\hline & Young modulus $(\mathrm{MPa})$ & Poisson's ratio \\
\hline Cancellous bone & 1,370 & 0.3 \\
\hline Cortical bone & 13,700 & 0.3 \\
\hline Ti cp & 11,000 & 0.28 \\
\hline Titanium alloy & 11,000 & 0.33 \\
\hline
\end{tabular}

The control group had 5 regular implants, arranged in parallel; the regular implants group had 3 regular implants, arranged in parallel; the distally tilted regular implants (DTRI) group had 3 regular implants, with the distal implants tilted 30 degrees; and the wide implants group had 5 wide implants, arranged in parallel. Regular and wide implants measured 3.75 and $5.0 \mathrm{~mm}$ in diameter, respectively. The bar was the same for all groups. The cantilever extension was $15 \mathrm{~mm}$ for the control, regular implants, and wide implants groups. The DTRI group had a shorter cantilever extension due to inclination of the distal implants.

FE models were obtained by importing the model into a mechanical simulation software package (ANSYS Workbench 11, Ansys Inc., Canonsburg, USA). All materials used in this study were considered to be isotropic, homogeneous, and linearly elastic. The elastic properties used (Table 1) were taken from the literature. ${ }^{13-16}$

Convergence tests, with $6 \%$ confidence levels, were performed to guarantee that the results were not influenced by the FE mesh. The implant thread was removed to reduce the number of elements. The elements used in the mesh were tetrahedral with 10 nodes. The final mesh presented an element size of $0.5 \mathrm{~mm}$. The number of elements and nodes generated in the FE models varied within the groups:

- control: 454,375 and 754,763;

- regular implants: 297,236 and 502,513;

- DTRI: 307,877 and 517,612;

- wide implants: 299,260 and 505,617, respectively).

Arrangements of the investigated models are presented in Figure 1. 
Figure 1 - Three-dimensional model configurations.
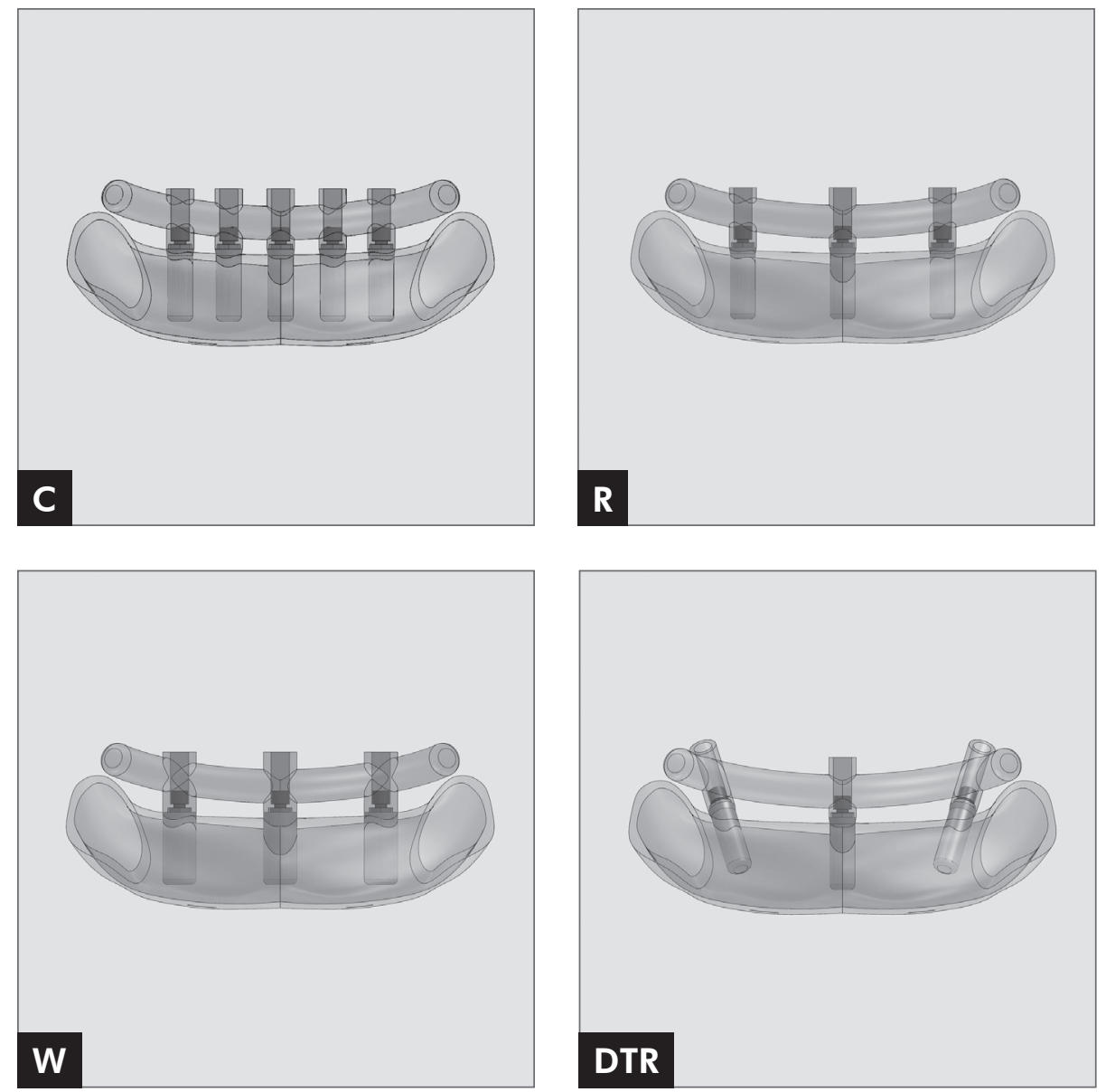

Table 2 - Maximum principal stresses (MPa), microstrain values, and percentage of variance in relation to the $C$ group in the peri-implant bone.

\begin{tabular}{|c|c|c|c|c|c|c|}
\hline \multirow[t]{2}{*}{ Group } & \multicolumn{2}{|c|}{ Left distal implant } & \multicolumn{2}{|c|}{ Medial Implant } & \multicolumn{2}{|c|}{ Right distal implant (loaded) } \\
\hline & $\begin{array}{l}\text { Maximum principal } \\
\text { stresses }\end{array}$ & Microstrain & $\begin{array}{l}\text { Maximum principal } \\
\text { stresses }\end{array}$ & Microstrain & $\begin{array}{l}\text { Maximum principal } \\
\text { stresses }\end{array}$ & Microstrain \\
\hline C & 3.445 & 221.33 & 3.53 & 222.70 & 5.27 & 952.34 \\
\hline R & 4.02 (+16.6\%) & $251.34(+13.5 \%)$ & $7.57(+114.2 \%)$ & $501.55(+125.2 \%)$ & $11.12(+111.0 \%)$ & $928.64(-2.4 \%)$ \\
\hline W & $3.38 \quad(-1.6 \%)$ & $215.16 \quad(-2.7 \%)$ & $3.48 \quad(-1.5 \%)$ & $245.67 \quad(+10.3 \%)$ & $4.64 \quad(-11.8 \%)$ & $931.49(-2.1 \%)$ \\
\hline DTR & $(6.9 \%)$ & $227.56 \quad(+2.8 \%)$ & $6.70 \quad(+89.5 \%)$ & $466.54(+109.4 \%)$ & $(+12.7 \%)$ & $889.79(-6.5 \%)$ \\
\hline
\end{tabular}

Constraint conditions for the displacement were applied to the mandible base. Loads were applied unilaterally in the right cantilever $(50 \mathrm{~N})$. Data for von Mises stresses for implants, screws, and frameworks, and the maximum principal stresses (MPS) and microstrain for peri-implant bone were analyzed, reproduced numerically, color-coded, and compared among the groups.

\section{Results}

The MPS and microstrain values on the periimplant bone are described in Table 2. The wide implants group showed the highest decrease in MPS $(-11.8 \%)$ in the peri-implant bone of the loaded implant compared to the control group. The regular implants group presented the highest increase in the medial implant (114.2\%). The control group 
presented the highest stress value in the right distal implant (loaded; $62.41 \mathrm{MPa}$ ), whereas the regular implants group presented a slight decrease $(-0.5 \%)$ and the DTRI $(-27.2 \%)$ and wide implants $(-41.2 \%)$ groups had larger reductions in stress values.

In all analyzed models, stresses were concentrated in the cortical bone near the implant platform. The highest stress values were observed adjacent to the load application. The stress values decreased progressively as the components were located further from the load (Table 3 and Figure 2).

As shown in Table 4, the wide implants group presented a reduction in the stress values in all screws compared to the control group. However, both arrangements (parallel and tilted implants) with 3 implants of regular diameter presented an increase in stress values, except for the right distal screw (loaded) in the DTRI group.

The stress distribution in the framework is presented in Table 5. The DTRI group presented the lowest and the regular implants group the highest value of stress.

\section{Discussion}

The hypothesis of this study was partially confirmed. Reducing the number of implants from 5

Table 3 - von Mises stresses $(\mathrm{MPa})$ and percentage of variance in relation to the $\mathrm{C}$ group in the implants.

\begin{tabular}{c|c|c|c}
\hline & Left distal implant & Medial implant & Right distal implant (loaded) \\
\hline$C$ & 5.16 & 11.27 & 62.41 \\
\hline$R$ & $9.00(+74.3 \%)$ & $23.17(+105.5 \%)$ & $62.07 \quad(-0.5 \%)$ \\
\hline$W$ & $5.94(+15.0 \%)$ & $14.53(+28.8 \%)$ & $36.66(-41.2 \%)$ \\
\hline DTR & $8.89(+72.3 \%)$ & $18.14 \quad(+60.9 \%)$ & $45.42 \quad(-27.2 \%)$ \\
\hline
\end{tabular}

Figure 2 - Stress distribution in the prosthetic components on the different groups.
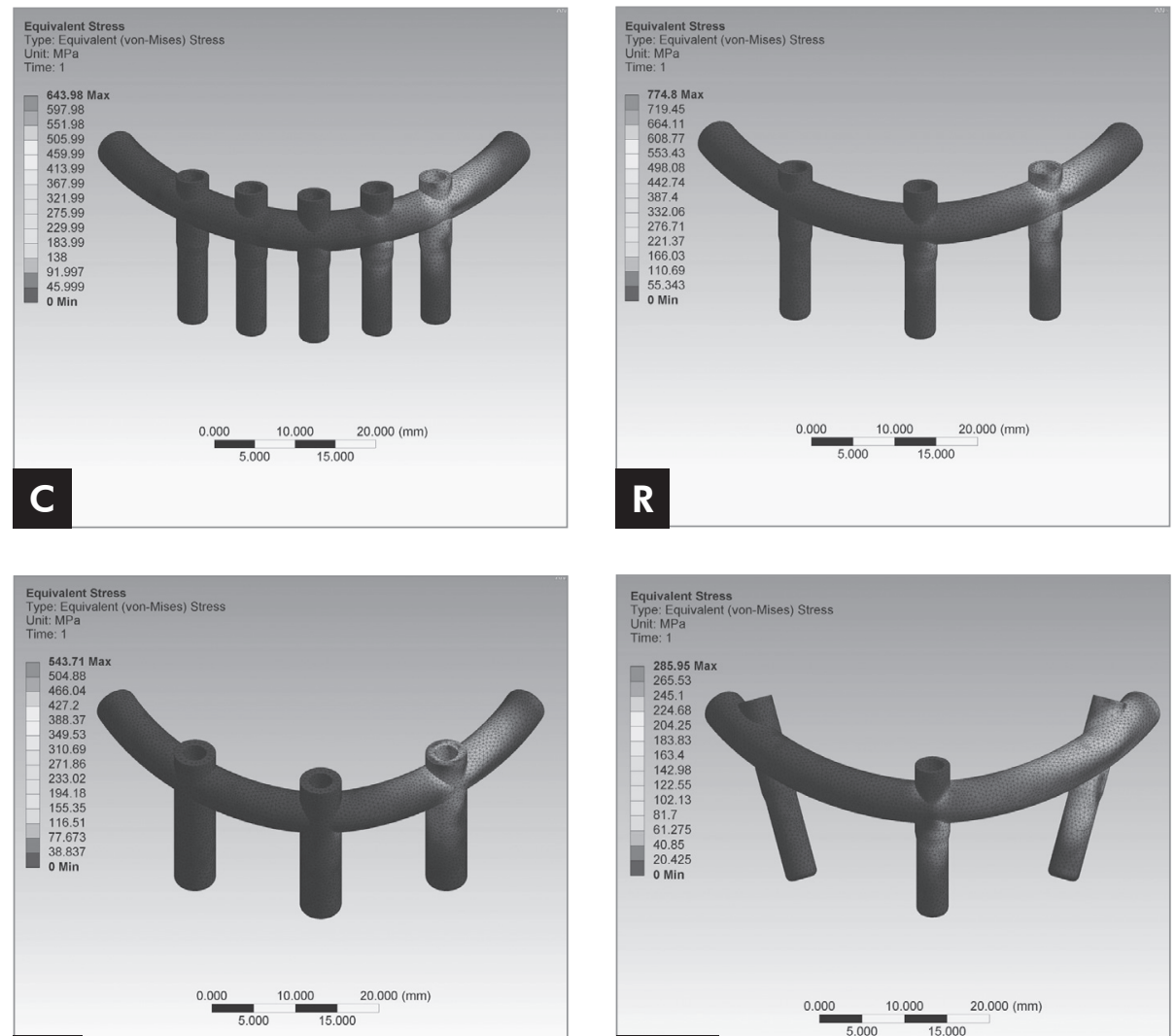

W

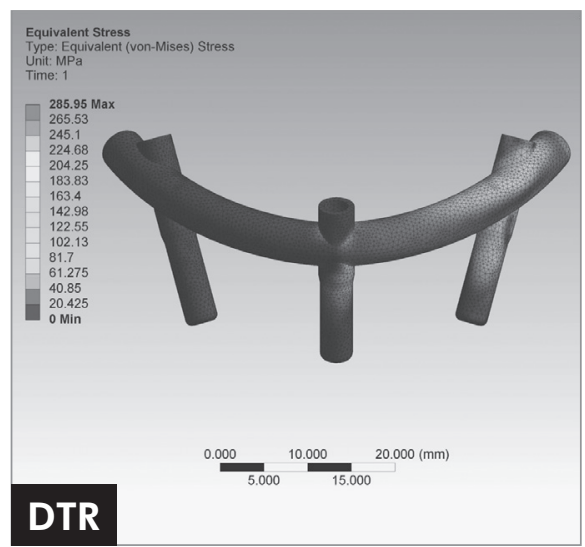


Table 4 - von Mises (MPa) stresses and percentage of variance in relation to the $\mathrm{C}$ group in the distal and medial screws.

\begin{tabular}{c|c|c|c}
\hline & Left distal screw & Medial screw & Right distal screw (loaded) \\
\hline$C$ & 1.91 & 5.81 & 19.136 \\
\hline$R$ & $2.84(+48.4 \%)$ & $11.45(+97.1 \%)$ & $21.69(+13.3 \%)$ \\
\hline W & $1.17(-38.6 \%)$ & $4.97(-14.3 \%)$ & $15.04(-21.3 \%)$ \\
\hline DTR & $2.74(+43.1 \%)$ & $9.55(+64.4 \%)$ & $18.30(-4.3 \%)$ \\
\hline
\end{tabular}

(control group) to 3 (regular implants group) increased the stress in the peri-implant bone, implants (except the loaded implant), screws, and framework. However, the stress values were reduced for the wide implants group, which had fewer implants of greater diameter compared to the control group. These results corroborate the findings proposed by the Novum concept. ${ }^{17}$ Thus, although high clinical success rates have been obtained for mandibular implant-supported rehabilitations using 3 implants, the use of regular implants in this setting can possibly overload the components, resulting in higher rates of screw loosening, which leads to a greater need for more follow-up appointments.

The possibility of reducing the number of implants was based on the distribution of load between them. According to Duyck et al. ${ }^{18}$ the distal and central implants receive the greatest loads, regardless of the number of implants between them or the total number of implants. Such findings suggest that it is not necessary to use a large number of implants to support a mandibular fixed implantsupported prosthesis. ${ }^{9}$ In the present study, the regular implants group showed the highest stress values in the peri-implant bone. The stress in the peri-implant bone was higher as it approached the region of load application, whereas the unloaded distal implant presented the lowest stress values for all groups.

When the DTRI group was compared to the regular implants group, the tilted implants were even more important in decreasing stress on the loaded region. These findings confirm previous evaluations of mandibular fixed rehabilitations supported by 4 implants. Those previous studies found a stress reduction when the distal implants were tilted about $30^{\circ}-45^{\circ}$, due to a reduction in the lever arm and largest cortical bone area for stress distribu-
Table 5 - von Mises (MPa) stresses and percentage of variance in relation to the $\mathrm{C}$ group in the framework.

\begin{tabular}{c|c}
\hline & Framework \\
\hline C & 128.80 \\
\hline$R$ & $154.96(+20.3 \%)$ \\
\hline$W$ & $108.74(-15.5 \%)$ \\
\hline DTR & $57.19(-55.6 \%)$ \\
\hline
\end{tabular}

tion. ${ }^{4,6,7,12}$ Nevertheless, the DTRI group presented higher levels of stresses in the peri-implant area of the loaded side when compared to the control and wide implants groups. In the screws and framework, components next to the loaded region presented higher stress values than the distant ones. The regular implants group showed the highest values for both screws and framework. The DTRI group presented increased stress values in the unloaded screws (medial and left distal), but the loaded screws had decreased stress values compared to the control group. The reduction in the number of implants could be a risk factor facilitating screw loosening or fracture. ${ }^{18} \mathrm{~A}$ decrease in the number of implants with maintenance of the implant diameter led to a relevant increase in stress in the prosthetic components. Similar studies evaluating the quantity and arrangement of implants did not evaluate prosthetic components. ${ }^{7,12}$ Thus, there are few data to allow comparisons.

The unloaded implants and screws of the DTRI group showed increased stress values compared to the control group, perhaps due to the reduction in the number of the implants and the dissipation of stress in the entire system. Although high stress concentrations could lead to long-term fatigue failure, ${ }^{8,9}$ the increased stress values were still lower than those observed in the regular implants group. 
The increase in diameter is more relevant to increasing the peri-implant bone surface than the implant length. Larger peri-implant bone surface areas provide greater dissipation of stresses when a prosthesis is loaded. In some cases, this situation can compensate for a reduced number of implants in prosthodontic rehabilitations. ${ }^{19-21}$ Similarly, in this study, a reduction in the number of implants associated with an increase in the implant diameter did not influence the stress and strain values in the peri-implant bone compared to the control group.

The wide implants group presented higher stress values in the unloaded implants compared to the control group. However, these stresses are probably supported by the implants due to their increased diameter and, as a result, the fatigue resistance is likely increased compared to regular implants. ${ }^{22} \mathrm{~A}$ comparison of the stress values of the wide implants group with the other groups with 3 implants showed more relevant results, indicating a better stress distribution.

These findings agree with the initial concerns reported in the development of the Novum system ${ }^{\circledR}$, which advocated a reduction in the number and an increase in the diameter of implants. ${ }^{3}$ The results also reinforce the hypothesis that failures observed in the Novum system ${ }^{\circledast}$ were possibly caused by inadequate implant positioning during the surgical procedures, due to the difficulty in obtaining a passive fit with prefabricated metallic frameworks. ${ }^{8}$ The use of wide implants in the anterior region of the mandible is a limitation on the prescription of this technique, due to the reduced bone quantity in this region. Also, the presence of type I cortical bone requires a change in the bone milling protocol and increases the risk of tissue necrosis.

In all arrangements with 3 implants, the microstrain values were below levels considered harmful to bone. ${ }^{23}$ The load applied in this test simulated a conventional denture as antagonist. If the load applied were to be increased, as is the case with opposing natural dentition or implant rehabilitations, then the stress values and strains would be more harmful to the system, possibly compromising the longevity of the therapy. Some studies have shown that the use of 3 implants in an edentulous mandible to support a fixed prosthesis is viable, and that the stresses generated during function are not sufficient to damage the osseointegration. However, these stresses can still be detrimental to the long-term success of the rehabilitation. ${ }^{8,9}$

Although UCLA abutments were used in the present study, micro-units are the most commonly used abutments in clinical application. However, micro-units are more expensive than UCLA abutments. Because the authors sought to evaluate the biomechanics of various implant distributions, UCLA abutments were used to minimize the costs. Use of FE analysis helps to overcome some traditional experimental methodological limitations, by offering information about the biomechanics of a given situation. ${ }^{13-16}$ However, FE analysis presents some limitations due to simplifications made in the analysis. The assumption of homogeneous, isotropic, and linearly elastic properties for the bone is common in the literature, ${ }^{13-16}$ but could affect the reliability of the results. Another limitation was the removal of the implant threads. Although a previous study observed that the threads influence the biomechanical behavior, ${ }^{24}$ their removal was useful for reducing the number of elements and nodes of the FE model. These aspects are limiting factors when the FE analysis comprises a large model with many components.

In this study, conventional rehabilitation with 5 implants did not present the best results for the prosthetic components and peri-implant bone. The use of 3 wide implants showed the lowest stress values for components and peri-implant bone, with the exception of the framework. Further studies using implant threads, nonlinear conditions, and different methodologies, such as experimental studies and clinical trials, should be performed to verify the effects of such arrangements on the longevity of this type of rehabilitation.

\section{Conclusion}

The arrangement of 3 regular implants presented the highest stress values in all evaluated regions. The use of 3 wide implants presented lower stress values compared to the standard 5-implant technique. 


\section{References}

1. Branemark PI, Svensson B, van Steenberghe D. Ten-year survival rates of fixed prostheses on four or six implants ad modum Branemark in full edentulism. Clin Oral Implants Res. 1995 Dec;6(4):227-31.

2. Mendonca DB, Prado MM, Mendes FA, Borges TF, Mendonca G, Prado CJ, et al. Comparison of masticatory function between subjects with three types of dentition. Int J Prosthodont. 2009 Jul-Aug;22(4):399-404.

3. Branemark PI, Engstrand P, Ohrnell LO, Grondahl K, Nilsson P, Hagberg K, et al. Branemark Novum: a new treatment concept for rehabilitation of the edentulous mandible. Preliminary results from a prospective clinical follow-up study. Clin Implant Dent Relat Res. 1999;1(1):2-16.

4. Begg T, Geerts GA, Gryzagoridis J. Stress patterns around distal angled implants in the all-on-four concept configuration. Int J Oral Maxillofac Implants. 2009 Jul-Aug;24(4):663-71.

5. Takahashi T, Shimamura I, Sakurai K. Influence of number and inclination angle of implants on stress distribution in mandibular cortical bone with All-on-4 Concept. J Prosthodont Res. 2010 Oct;54(4):179-84.

6. Kim KS, Kim YL, Bae JM, Cho HW. Biomechanical comparison of axial and tilted implants for mandibular full-arch fixed prostheses. Int J Oral Maxillofac Implants. 2011 SepOct;26(5):976-84.

7. Fazi G, Tellini S, Vangi D, Branchi R. Three-dimensional finite element analysis of different implant configurations for a mandibular fixed prosthesis. Int J Oral Maxillofac Implants. 2011 Jul-Aug;26(4):752-9.

8. Hatano N, Yamaguchi M, Yaita T, Ishibashi T, Sennerby L. New approach for immediate prosthetic rehabilitation of the edentulous mandible with three implants: a retrospective study. Clin Oral Implants Res. 2011 Nov;22(11):1265-9.

9. Rivaldo EG, Montagner A, Nary H, da Fontoura Frasca LC, Branemark PI. Assessment of rehabilitation in edentulous patients treated with an immediately loaded complete fixed mandibular prosthesis supported by three implants. Int J Oral Maxillofac Implants. 2012 May-Jun;27(3):695-702.

10. Engstrand P, Grondahl K, Ohrnell LO, Nilsson P, Nannmark $\mathrm{U}$, Branemark PI. Prospective follow-up study of 95 patients with edentulous mandibles treated according to the Branemark Novum concept. Clin Implant Dent Relat Res. 2003 May;5(1):3-10.

11. Krekmanov L, Kahn M, Rangert B, Lindstrom H. Tilting of posterior mandibular and maxillary implants for improved prosthesis support. Int J Oral Maxillofac Implants. 2000 MayJun;15(3):405-14.

12. Bevilacqua M, Tealdo T, Pera F, Menini M, Mossolov A, Drago C, et al. Three-dimensional finite element analysis of load transmission using different implant inclinations and cantilever lengths. Int J Prosthodont. 2008 Nov-Dec;21(6):539-42.

13. Dos Santos MB, Bacchi A, Consani RL, Mesquita MF. Influence of thickness and area of reline on the stress distribution in peri-implant bone during the healing period: a three-dimensional finite element analysis. Gen Dent. 2012 JulAug;60(4):e231-6.

14. Dos Santos MB, Consani RL, Mesquita MF. Influence of different soft liners on stress distribution in peri-implant bone tissue during healing period. A 3-D finite element analysis. J Oral Implantol. 2011 Jul 18. Epub ahead of print.

15. Dos Santos MB, Silva Neto JP, Consani RL, Mesquita MF. Three-dimensional finite element analysis of stress distribution in peri-implant bone with relined dentures and different heights of healing caps. J Oral Rehabil. 2011 Sep;38(9):691-6.

16. Spazzin AO, Dos Santos MB, Correr-Sobrinho L, Consani RL, Mesquita MF. Effects of horizontal misfit and bar framework material on the stress distribution of an overdenture-retaining bar system: a 3D finite element analysis. J Prosthodont. 2011 Oct;20(7):517-22.

17. Branemark PI. The Branemark Novum Protocol for same-day teeth. A global perspective. Berlin: Quintessence; 2000. 166 p.

18. Duyck J, Van Oosterwyck H, Vander Sloten J, De Cooman M, Puers R, Naert I. Magnitude and distribution of occlusal forces on oral implants supporting fixed prostheses: an in vivo study. Clin Oral Implants Res. 2000 Oct;11(5):465-75.

19. Himmlova L, Dostalova T, Kacovsky A, Konvickova S. Influence of implant length and diameter on stress distribution: a finite element analysis. J Prosthet Dent. 2004 Jan;91(1):20-5.

20. Mahon JM, Norling BK, Phoenix RD. Effect of varying fixture width on stress and strain distribution associated with an implant stack system. Implant Dent. 2000 Winter;9(4):310-20.

21. Renouard F, Nisand D. Impact of implant length and diameter on survival rates. Clin Oral Implants Res. 2006 Oct;17 Suppl 2:35-51.

22. Friberg B, Ekestubbe A, Sennerby L. Clinical outcome of Branemark System implants of various diameters: a retrospective study. Int J Oral Maxillofac Implants. 2002 SepOct;17(5):671-7.

23. Wiskott HW, Belser UC. Lack of integration of smooth titanium surfaces: a working hypothesis based on strains generated in the surrounding bone. Clin Oral Implants Res. 1999 Dec;10(6):429-44.

24. Huang HL, Chang CH, Hsu JT, Fallgatter AM, Ko CC. Comparison of implant body designs and threaded designs of dental implants: a 3-dimensional finite element analysis. Int J Oral Maxillofac Implants. 2007 Jul-Aug;22(4):551-62. 